Hydrology and Earth System Sciences, 8(2), 247-255 (2004) (C) EGU

\title{
Comparison of three updating schemes using artificial neural network in flow forecasting
}

\author{
Lihua Xiong ${ }^{1}$, Kieran M. O'Connor ${ }^{2}$ and Shenglian Guo ${ }^{1}$ \\ ${ }^{1}$ State Key Laboratory of Water Resources and Hydropower Engineering Science, Wuhan University, Wuhan, 430072, China \\ ${ }^{2}$ Department of Engineering Hydrology, National University of Ireland, Galway, Ireland \\ E-mail for corresponding author: lhxiong@public.wh.hb.cn
}

\begin{abstract}
Three updating schemes using artificial neural network (ANN) in flow forecasting are compared in terms of model efficiency. The first is the ANN model in the simulation mode plus an autoregressive (AR) model. For the ANN model in the simulation model, the input includes the observed rainfall and the previously estimated discharges, while the AR model is used to forecast the flow simulation errors of the ANN model. The second one is the ANN model in the updating mode, i.e. the ANN model uses the observed discharge directly together with the observed rainfall as the input. In this scheme, the weights of the ANN model are obtained by optimisation and then kept fixed in the procedure of flow forecasting. The third one is also the ANN model in the updating mode; however, the weights of the ANN model are no longer fixed but updated at each time step by the backpropagation method using the latest forecast error of the ANN model. These three updating schemes are tested for flow forecasting on ten catchments and it is found that the third updating scheme is more effective than the other two in terms of their efficiency in flow forecasting. Moreover, compared to the first updating scheme, the third scheme is more parsimonious in terms of the number of parameters, since the latter does not need any additional correction model. In conclusion, this paper recommends the ANN model with the backpropagation method, which updates the weights of ANN at each time step according to the latest forecast error, for use in real-time flow forecasting.
\end{abstract}

Keywords: artificial neural network (ANN), updating, flow forecasting, backpropagation method

\section{Introduction}

The application of the artificial neural network (ANN) in hydrology, especially in rainfall-runoff modelling, has become very popular in recent years because the ANN models have the ability to approximate any continuous (nonlinear) relationships (Hsu et al., 1995; Imrie et al., 2000; Dekker et al., 2001). Such applications can be found in many papers published recently (French et al., 1992; Zhu et al., 1994; Hsu et al.,1995; Smith and Eli, 1995; Minns and Hall, 1996; Shamseldin, 1997; Campolo et al., 1999; Gautam et al., 2000; Imrie et al., 2000; Chang and Chen, 2001; Shamseldin and O'Connor, 2001; Xiong and O'Connor, 2002; Campolo et al., 2003; Wilby et al., 2003; Jain et al., 2004). All these works have demonstrated that ANN models are indeed very flexible and sufficiently efficient to simulate the rainfall-runoff processes.
In most cases, the ANN models used in hydrology are regarded as black-box models that cannot provide any physically realistic structure and parameters to represent the hydrological processes in catchments, even though they are capable of identifying complex non-linear relationships between rainfall and runoff time series (Hsu et al., 1995). In this sense, the ANN model is not an alternative to the conceptual or the physically-based distributed model for the analysis of the physical mechanisms of the hydrological processes in the catchment. However, recent works (Wilby et al., 2003; Jain et al., 2004) have demonstrated that a trained ANN rainfall-runoff model can, to some degree, reflect certain physical processes in catchments.

When any rainfall-runoff model, including an ANN model, is intended for use in a real-time forecasting situation, it will be associated with an explicit or implicit updating 
procedure whereby, at the time of making the forecast, errors already observed in recent forecasts will be used to modify the forecast. These errors result from inadequacies in the model structure, incorrect estimation of the model parameters, errors in the data or, indeed, the absence of any consistent relationship in the data (Kachroo and Liang, 1992). Observation of the structure of the error persistence can provide the basis for an updating procedure. Although the ultimate objective of the updating procedure is to improve flow forecasts, the items used in the updating procedure can be one or more of the following: inputs: rainfall, state variables such as soil moisture content, model parameters, or outputs such as the simulated flow (Georgakakos and Smith, 1990; WMO, 1992).

In this paper, the three updating schemes using ANN in real-time flow forecasting are compared in terms of model efficiency. The paper is organised as follows. Firstly, the basic framework of employing an ANN in rainfall-runoff modelling is presented. Secondly, three updating schemes using an ANN in flow forecasting are described in detail. Finally, these three updating schemes using an ANN are applied to ten catchments and the results are compared.

\section{Artificial neural networks (ANNs)}

Many ANN structures have been proposed and explored for tasks such as recognition, learning, forecasting and controlling. Among these different structures, the multilayer feed forward networks have the best performance in the context of input-output function approximation (Haykin, 1994; Friedman and Kandel, 1999). As a matter of fact, almost all ANNs explored in rainfall-runoff modelling are multilayer feed forward networks (Campolo et al., 1999).

A typical multilayer neural network with a single hidden layer is illustrated in Fig. 1 (Friedman and Kandel, 1999). It has input nodes $\left\{X_{i}(p)\right\}_{i=1}^{n}$ (and a bias), hidden nodes $\left\{Z_{j}(p)\right\}_{j=1}^{l}$ (and a bias) and output nodes $\left\{Y_{k}(p)\right\}_{k=1}^{m}$, where $X, Z$, and $Y$ represent the input, hidden and output layer respectively, $n, l$, and $m$ represent the number of the nodes in each layer and $p$ denotes training pattern. The weights associated with the connections between the input and the hidden nodes are denoted by $v_{i j}, 0 \leq i \leq n, 1 \leq j \leq l$ and those between the hidden and the output nodes are denoted by $w_{j k}, 0 \leq j \leq l, 1 \leq k \leq m$.

For node $Z_{j}$ in the hidden layer (Fig.1), its effective income signal, denoted by $z_{-} i n_{j}$, is calculated as

$$
z_{-} \text {in }_{j}=v_{0 j}+\sum_{i=1}^{n} v_{i j} x_{i}, 1 \leq j \leq l
$$

where $x_{i}, 1 \leq i \leq n$, represents the input to each node in the input layer.

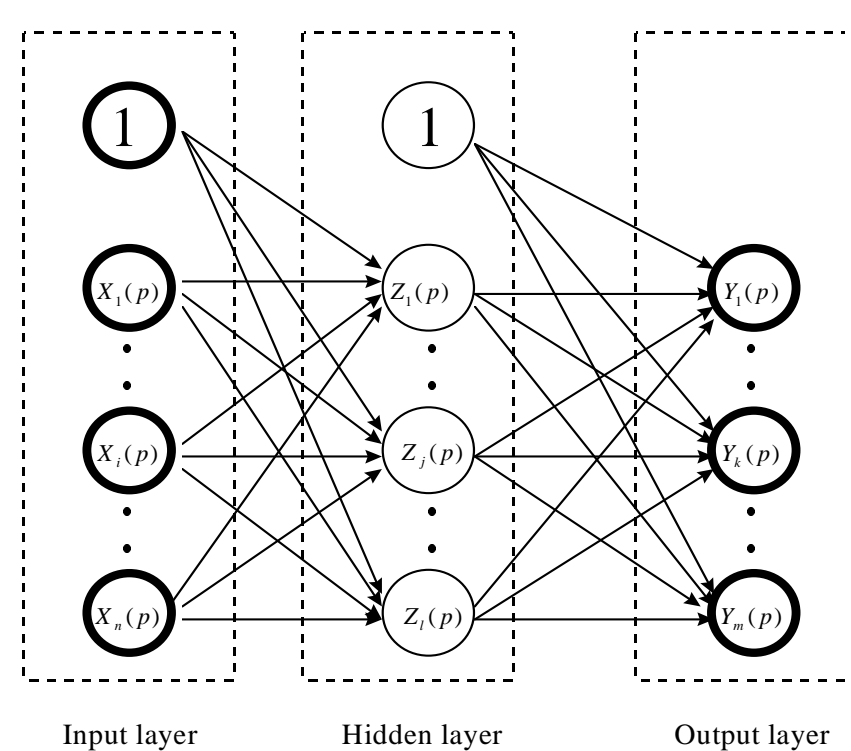

Fig. 1. A multilayer neural network with a single hidden layer

For node $Z_{j}$, its corresponding output signal, denoted by $z_{j}$, is obtained by using an activation function $f(x)$

$$
z_{j}=f\left(z_{-} i n_{j}\right), 1 \leq j \leq l
$$

The most widely used activation function is the sigmoid function (Haykin, 1994; Friedman and Kandel, 1999; Dekker et al., 2001). Among several different sigmoid functions, the one most often used for ANNs is the logistic function

$$
z_{j}=f\left(z_{-} i n_{j}\right)=\frac{1}{1+\exp \left(-\sigma \cdot z_{-} i n_{j}\right)}
$$

where $\sigma$ is an adjustable parameter used in the activation function $f(x)$.

Among the algorithms used to perform supervised training, the backpropagation method (Rumelhart et al., 1986) has emerged as the most widely used and successful algorithm for the design of the multilayer feed forward neural networks (Haykin, 1994) and in hydrology, the backpropagation method has already been used (French et al., 1992; Gautam et al., 2000; Wilby et al., 2003).

\section{Application of ANN in rainfall-runoff modelling}

As any catchment has a certain storage capacity, the runoff at its outlet is related not only to the current rainfall rate but also to the past rainfall and runoff situations. For a discrete lumped hydrological system, the rainfall-runoff relationship 


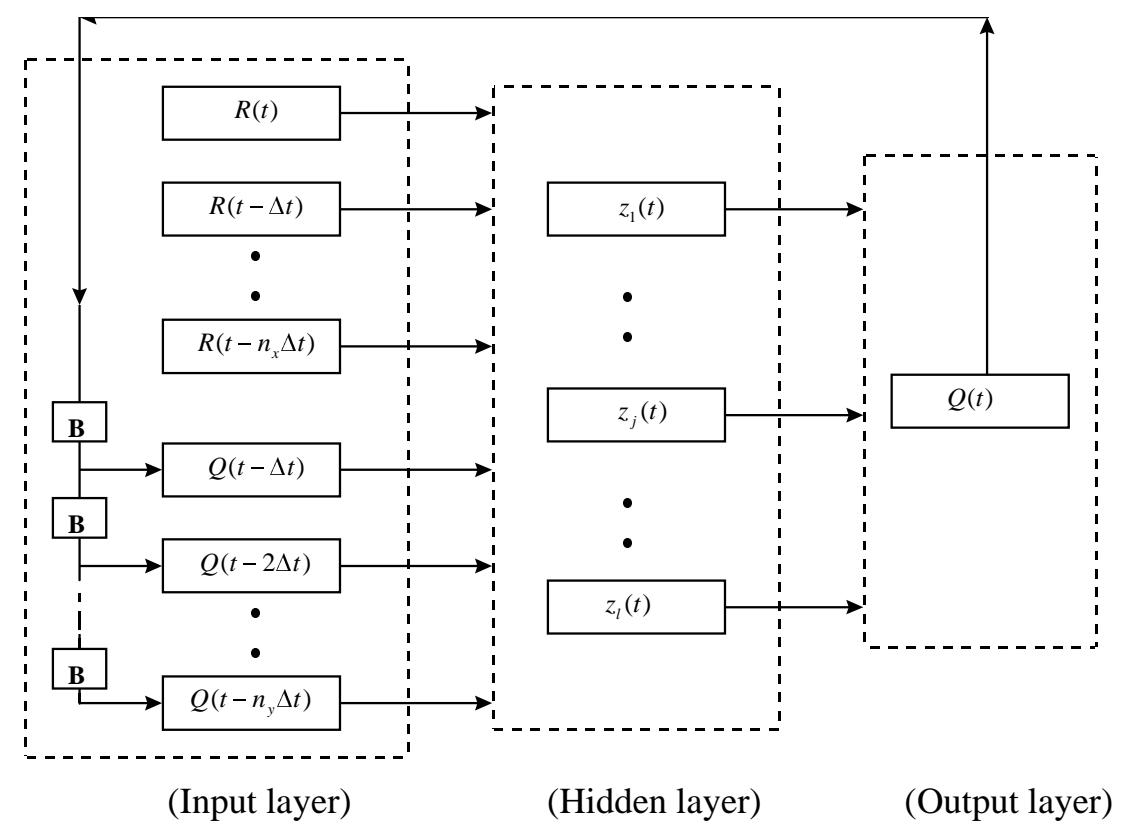

Fig.2. The block diagram of the ANN for rainfall-runoff modelling (where B is the unit delay operator or the backward shift operator, defined

$$
\text { by } \left.\boldsymbol{B}^{\mathrm{r}} Q(t)=Q(t-r \Delta t) \text { for } \mathrm{r}=0,1,2, \ldots\right)
$$

can be generally approximated as (Chow et al., 1988; Hsu et al., 1995)

$$
\begin{gathered}
Q(t)=F\left[R(t), R(t-\Delta t), \ldots, R\left(t-n_{x} \Delta t\right), Q(t-\Delta t),\right. \\
\left.\ldots Q\left(t-n_{y} \Delta t\right)\right]
\end{gathered}
$$

where $R$ represents rainfall, $Q$ is runoff at the outlet of the catchment, $F$ is the appropriate model structure (i.e. the mathematical functions), $\Delta t$ is the data sampling interval, and $n_{x}$ and $n_{y}$ are positive integers reflecting the memory length of the catchment. When the ANN is implemented to approximate the above relationship between the catchment average rainfall and runoff, there will be a number of $n_{x}+n_{y}+1$ nodes in the input layer, i.e. $n=n_{x}+n_{y}+1$, while there is only one node in the output, i.e. $m=1$. The corresponding ANN block diagram is plotted in Fig.2.

\section{Three updating schemes for real-time flow forecasting}

Three kinds of real-time flow forecasting schemes involving the ANN models are described in detail as follows.

\section{UPDATING SCHEME NO.1}

The first updating scheme is the ANN model in the simulation mode plus an AR model, which involves three steps. Firstly, use one of the global search methods (Duan et al., 1992) to find a set of optimum values for the weights in the ANN model, which are denoted by $v_{i j}^{o p t}, 0 \leq i \leq n$, $1 \leq j \leq l$ and $w_{j k}^{o p t}, 0 \leq j \leq l, 1 \leq k \leq m$. The inputs to the ANN model include the observed rainfall and the estimated discharge at the previous steps. In this paper, the selected optimisation algorithm is the Simplex method (Press et al., 1989). The estimated runoffs, denoted by $\hat{Q}(t)$, will be determined as a function of those optimum weights of the ANN, which is expressed as

$$
\begin{aligned}
\hat{Q}(t)=F_{A N N}\left[R(t), R(t-\Delta t), \ldots, R\left(t-n_{x} \Delta t\right),\right. & \left.\hat{Q}(t-\Delta t), \ldots \hat{Q}\left(t-n_{y} \Delta t\right) \mid v_{i j}^{o p t}, w_{j k}^{o p t}\right]
\end{aligned}
$$

Secondly, according to the simulation error series of the ANN model, an AR model is employed to estimate such errors

$$
\hat{e}(t)=a_{0}+a_{1} e(t-\Delta t)+a_{2} e(t-2 \Delta t)+\ldots+a_{s} e(t-s \Delta t)
$$

where $e$ represents the flow simulation errors of the ANN model, i.e. $e=Q-\hat{Q}, a_{0}$ is the regression constant term, $a_{1}, a_{2}, \ldots, a_{s}$ are the regression coefficients, $s$ is the order of the AR model, and $\hat{e}$ is the estimated error.

Finally, the updated forecast, denoted by $Q^{\prime}(t)$, is calculated by

$$
Q^{\prime}(t)=\hat{Q}(t)+\hat{e}(t)
$$

This first updating scheme, i.e. the ANN model in the simulation mode plus the AR model as the corrective model, 
is the most widely used paradigm in real time flow forecasting (Kachroo and Liang, 1992; WMO, 1992; Xiong and O'Connor, 2002).

\section{UPDATING SCHEME NO.2}

The second updating scheme is the ANN model in the updating mode using, rather than those simulated, the observed discharges, together with the observed rainfall, as the input. In this scheme, the weights of the ANN model are obtained by optimisation and then kept fixed in the procedure of flow forecasting. Mathematically, this updating scheme is expressed as

$$
\begin{array}{r}
Q^{\prime}(t)=F_{A N N}\left[R(t), R(t-\Delta t), \ldots, R\left(t-n_{x} \Delta t\right),\right. \\
\left.Q(t-\Delta t), \ldots Q\left(t-n_{y} \Delta t\right) \mid v_{i j}^{o p t}, w_{j k}^{o p t}\right]
\end{array}
$$

The optimised values of the weights $v_{i j}^{o p t}$ and $w_{j k}^{o p t}$ found in this scheme will be different from those in the first updating scheme because of the different inputs.

\section{UPDATING SCHEME NO.3}

The third updating scheme is the ANN model in the updating mode but the weights of the ANN model are no longer fixed but updated at each time step by the backpropagation method (Rumelhart et al., 1986) using the error in the latest forecast of the ANN model. These new weights are used to forecast the runoff at the next time step. Thus, it is no longer necessary to find a fixed set of the optimum values for the weights $v_{i j}, 0 \leq i \leq n, 1 \leq j \leq l$ and $w_{j k}, 0 \leq j \leq l, 1 \leq k \leq m$, but rather to find a set of optimum values just for the initial weights $v_{i j}^{(0)}$ and $w_{j k}^{(0)}$. This third real-time forecasting scheme is explained mathematically as follows.

At time step $(t-2 \Delta t)$, assume that updated weights for the ANN model have been obtained by the backpropagation method for the next time step $(t-\Delta t)$; these updated weights are denoted $v_{i j}(t-\Delta t)$ and $w_{j k}(t-\Delta t)$. The flow forecast for $Q(t-\Delta t)$, which is $Q^{\prime}(t-\Delta t)$, is then calculated as:

$$
\begin{array}{r}
Q^{\prime}(t-\Delta t)=F_{A N N}\left[R(t-\Delta t), R(t-2 \Delta t), \ldots, R\left(t-\left(n_{x}+1\right) \Delta t\right),\right. \\
\left.Q(t-2 \Delta t), \ldots Q\left(t-\left(n_{y}+1\right) \Delta t\right) \mid v_{i j}(t-\Delta t), w_{j k}(t-\Delta t)\right]
\end{array}
$$

At the next time step, i.e. the time step $(t-\Delta t)$, the measurement of the discharge, $Q(t-\Delta t)$ is now available. According to the error in the latest forecast i.e. $Q(t-\Delta t)-Q^{\prime}(t-\Delta t)$, the weights of the ANN will be updated again as follows:

$$
\begin{array}{r}
v_{i j}(t)=v_{i j}(t-\Delta t)+\Delta v_{i j}(t-\Delta t), 1 \leq i \leq n, 1 \leq j \leq l \\
w_{j k}(t)=w_{j k}(t-\Delta t)+\Delta w_{j k}(t-\Delta t), 1 \leq j \leq l, 1 \leq k \leq m
\end{array}
$$

where $v_{i j}(t)$ and $w_{j k}(t)$ are the updated weights to be used for flow forecasting at the time step $t, \Delta v_{i j}(t-\Delta t)$ and $\Delta w_{j k}(t-\Delta t)$ are the weight correction terms, which are determined according to the forecast error $Q(t-\Delta t)-Q^{\prime}(t-\Delta t)$.

With the weights $v_{i j}(t)$ and $w_{j k}(t)$ now obtained, the flow forecast of the ANN for the time step $t$, is expressed as

$$
\begin{aligned}
Q^{\prime}(t)=F_{A N N}\left[R(t), R(t-\Delta t), \ldots, R\left(t-n_{x} \Delta t\right)\right. & \\
& \left.Q(t-\Delta t), \ldots Q\left(t-n_{y} \Delta t\right) \mid v_{i j}(t), w_{j k}(t)\right]
\end{aligned}
$$

where $Q^{\prime}(t)$ is the real time forecast for the flow discharge $Q(t)$.

At time step $t$, when the observed discharge $Q(t)$ is available, then the weights will be updated again for forecasting at the next time step $(t+\Delta t)$. The above procedure will be repeated as time proceeds.

In this third updating scheme, the initial values for the weights $v_{i j}^{(0)}$ and $w_{j k}^{(0)}$, in theory, can be determined by any optimisation method. For example, the optimised weight values $v_{i j}^{o p t}(0 \leq i \leq n, 1 \leq j \leq l)$ and $w_{j k}^{o p t}(0 \leq j \leq l$, $1 \leq k \leq m$ ), used in the second updating scheme, may be taken as the initial weights $v_{i j}^{(0)}$ and $w_{j k}^{(0)}$.

\section{Applications of the ANN in flow forecasting}

\section{THE STUDY CATCHMENT}

Ten catchments have been selected to test the efficiency of the three updating schemes. Basic information on these ten catchments and the data used are in Table 1.

\section{THE MODEL EFFICIENCY CRITERIA}

The main model criterion for assessing the ANN model efficiency is chosen to be the widely used Nash-Sutcliffe model efficiency index $R^{2}$ (Nash and Sutcliffe, 1970). The second index to assess the model performance is the index of volumetric fit $(I V F)$, which is defined as the ratio of the simulated runoff volume to that observed. In fact, many different model forecast performance criteria are available (e.g. WMO, 1975; ASCE, 1993; Legates and McCabe, 1999; Beran, 1999), corresponding to different objectives and different perspectives but the Nash-Sutcliffe index $R^{2}$ is probably the most widely used and perhaps the most 
Table 1. Hydrological conditions of the ten catchments

\begin{tabular}{llllll}
\hline Name & Country & Area $\left(\mathrm{km}^{2}\right)$ & Topography & Climate & $\begin{array}{l}\text { Data for calibration and } \\
\text { verification (days) }\end{array}$ \\
\hline Brosna & Ireland & 1,207 & Flat & Temperate & $2922 / 730$ \\
Chu & Vietnam & 2,090 & Hilly & Monsoon & $2922 / 730$ \\
Ihimbu & Tanzania & 2,480 & Hilly & Semi-arid & $1096 / 730$ \\
Kizu & Japan & 1,445 & Hilly & Humid & $1096 / 730$ \\
Shiquan-1 & China & 23,805 & Mixed & Temperate & $2191 / 731$ \\
Shiquan-3 & China & 3,092 & Mixed & Temperate & $2191 / 731$ \\
Sunkosi-1 & Nepal & 18,000 & Hilly & Cold & $2192 / 730$ \\
Qingjiang & China & 15,300 & Hilly & Humid & $1461 / 365$ \\
Wolomi Brook & Australia & 1,580 & Mixed & Arid & $1461 / 365$ \\
Yanbian & China & 2,350 & Flat & Semi-arid & $2191 / 731$ \\
\hline
\end{tabular}

important index for assessing the flood forecasting efficiency. As Legates and McCabe (1999) and others have reported, the Nash-Sutcliffe index $R^{2}$ is rather crude; it is oversensitive to extreme values, because of the squared differences in the definition, yet is insensitive to additive and proportional differences between model predictions and observations. Despite its recognised limitations, the $R^{2}$ criterion has been adopted as the main forecasting efficiency index in this study, supplemented by the index of volumetric fit and also by subjective visual comparisons of the simulated and observed discharge hydrographs.

\section{THE RESULTS}

The simulations of the three different updating schemes are listed in Table 2. Those of the first updating scheme include those for the simulation mode as well as for the updating mode. In the simulation model, using values of the weights determined by the Simplex optimisation method, the average efficiency, $\overline{R^{2}}$, is $78.64 \%$ in the calibration period and $66.17 \%$ in the verification period. Using the AR model to simulate the error series of the ANN improves the value of $\overline{R^{2}}$ to $90.11 \%$ in the calibration period and $82.69 \%$ in the verification period. For the second updating scheme, the average model efficiency value, $\overrightarrow{R^{2}}$, is $89.63 \%$ in the calibration period and $80.55 \%$ in the verification period. The third updating scheme, using the backpropagation method to update the weights at each time step, gives $\overline{R^{2}}$ as $92.95 \%$ in the calibration period and $85.07 \%$ in the verification period. It should be noted that the initial values for the weights in the third updating scheme are also found by the backpropagation training method. The simulated and observed hydrographs for the Sunkosi-1 catchment are in Fig. 3 and for the Yanbian catchment in Fig.4.

The above results show that all three updating schemes are more efficient than the ANN model in the simulation mode. The improvement in the average model efficiency value $\overline{R^{2}}$ by the first updating scheme is $11.47 \%$ in the calibration period and $16.52 \%$ in the verification period. In the second scheme, the increase in $\overline{R^{2}}$ is $10.99 \%$ in the calibration period and $14.38 \%$ in the verification period. For the third updating scheme, the increases in $R^{2}$ are $14.32 \%$ and $18.90 \%$ respectively, both of which are the largest increases.

In terms of the improvement of the updating schemes on the average model efficiency value $\overline{R^{2}}$, the first updating scheme is the least effective, probably because the parameters used in both the ANN (the substantive model) and the AR model (the correction model) are fixed and not very flexible in reflecting the time-varying characteristics of the hydrological processes in the catchment over rather long periods. The same is true for the second updating scheme. However, in the third updating scheme, the continuous updating of all weights in the ANN according to the forecast error at the previous time step enables the ANN model to track the time-variation characteristics of hydrological processes.

Furthermore, compared to the first updating scheme, the third updating scheme is more parsimonious in the number of parameters since it does not need any correction model, i.e. the AR model. This is because the ANN has the ability to self-adjust its weights (or parameters) according to new information, when the backpropagation method is used to train the ANN model.

\section{THE VARIATION OF THE WEIGHTS IN THE THIRD UPDATING SCHEME}

In the third updating scheme, the weights in the ANN model vary at each time step. As an example, the variation processes 
Table 2. The results of three real-time forecasting schemes using the ANN

\begin{tabular}{|c|c|c|c|c|c|c|c|c|c|c|c|c|c|}
\hline \multirow[t]{3}{*}{ Catchment } & \multirow{2}{*}{\multicolumn{5}{|c|}{ ANN structure }} & \multicolumn{4}{|c|}{ Scheme No.1 } & \multirow{2}{*}{\multicolumn{2}{|c|}{ Scheme No.2 }} & \multicolumn{2}{|c|}{ Scheme No.3 } \\
\hline & & & & & & \multicolumn{2}{|c|}{ simulation } & \multicolumn{2}{|c|}{ updating } & & & \multirow[b]{2}{*}{$R^{2}(\%)$} & \multirow[b]{2}{*}{ IVF } \\
\hline & $n_{x}$ & $n_{y}$ & $l$ & $m$ & & $R^{2}(\%)$ & $I V F$ & $R^{2}(\%)$ & $I V F$ & $R^{2}(\%)$ & $I V F$ & & \\
\hline \multirow[t]{2}{*}{ Brosna } & 1 & 3 & 3 & 1 & Cali. & 50.96 & 0.99 & 92.19 & 1.00 & 91.94 & 1.00 & 92.88 & 1.00 \\
\hline & & & & & Veri. & 40.55 & 0.98 & 93.64 & 1.00 & 93.29 & 1.00 & 93.33 & 1.00 \\
\hline \multirow[t]{2}{*}{ Chu } & 1 & 4 & 3 & 1 & Cali. & 78.25 & 1.07 & 80.93 & 1.00 & 75.98 & 1.06 & 85.50 & 1.05 \\
\hline & & & & & Veri. & 71.26 & 1.09 & 76.53 & 1.02 & 70.25 & 0.99 & 70.11 & 1.09 \\
\hline \multirow[t]{2}{*}{ Ihimbu } & 1 & 3 & 3 & 1 & Cali. & 65.81 & 1.03 & 95.54 & 1.00 & 96.18 & 1.00 & 95.78 & 1.00 \\
\hline & & & & & Veri. & 51.34 & 1.18 & 94.65 & 1.01 & 95.04 & 1.03 & 95.46 & 1.00 \\
\hline \multirow[t]{2}{*}{ Kizu } & 2 & 1 & 3 & 1 & Cali. & 89.37 & 1.01 & 92.31 & 1.00 & 91.39 & 1.02 & 93.50 & 1.02 \\
\hline & & & & & Veri. & 69.65 & 1.31 & 80.73 & 1.10 & 81.31 & 1.20 & 86.67 & 1.09 \\
\hline \multirow[t]{2}{*}{ Qingjiang } & 3 & 2 & 3 & 1 & Cali. & 88.45 & 1.01 & 90.68 & 1.00 & 91.66 & 1.00 & 92.00 & 1.00 \\
\hline & & & & & Veri. & 86.51 & 1.00 & 89.94 & 1.00 & 88.47 & 1.05 & 87.96 & 1.01 \\
\hline \multirow[t]{2}{*}{ Shiquan-1 } & 2 & 1 & 3 & 1 & Cali. & 82.14 & 1.08 & 87.01 & 1.00 & 82.54 & 1.04 & 91.53 & 1.00 \\
\hline & & & & & Veri. & 54.14 & 0.96 & 69.53 & 0.95 & 77.10 & 1.04 & 67.02 & 0.94 \\
\hline \multirow[t]{2}{*}{ Shiquan-3 } & 1 & 3 & 3 & 1 & Cali. & 86.07 & 1.04 & 88.28 & 1.00 & 88.50 & 1.07 & 91.93 & 1.05 \\
\hline & & & & & Veri. & 61.66 & 1.52 & 70.52 & 1.25 & 68.59 & 1.34 & 75.49 & 1.12 \\
\hline \multirow[t]{2}{*}{ Sunkosi-1 } & 1 & 2 & 2 & 1 & Cali. & 92.16 & 1.02 & 95.94 & 1.00 & 97.77 & 1.00 & 97.65 & 1.00 \\
\hline & & & & & Veri. & 82.14 & 0.78 & 97.35 & 0.96 & 98.60 & 0.97 & 98.07 & 1.00 \\
\hline Wolomi & 4 & 2 & 3 & 1 & Cali. & 72.02 & 0.95 & 87.15 & 1.00 & 89.02 & 0.80 & 95.03 & 1.11 \\
\hline Brook & & & & & Veri. & 65.82 & 1.56 & 64.67 & 1.15 & 43.51 & 1.05 & 85.19 & 0.92 \\
\hline \multirow[t]{2}{*}{ Yanbian } & 1 & 3 & 3 & 1 & Cali. & 81.18 & 1.09 & 91.08 & 1.00 & 91.28 & 1.00 & 93.68 & 1.00 \\
\hline & & & & & Veri. & 78.66 & 1.10 & 89.33 & 1.00 & 89.32 & 1.00 & 91.44 & 1.00 \\
\hline \multirow[t]{2}{*}{ Mean } & & & & & & 78.64 & 1.03 & 90.11 & 1.00 & 89.63 & 1.00 & 92.95 & 1.02 \\
\hline & & & & & & 66.17 & 1.15 & 82.69 & 1.04 & 80.55 & 1.07 & 85.07 & 1.02 \\
\hline
\end{tabular}

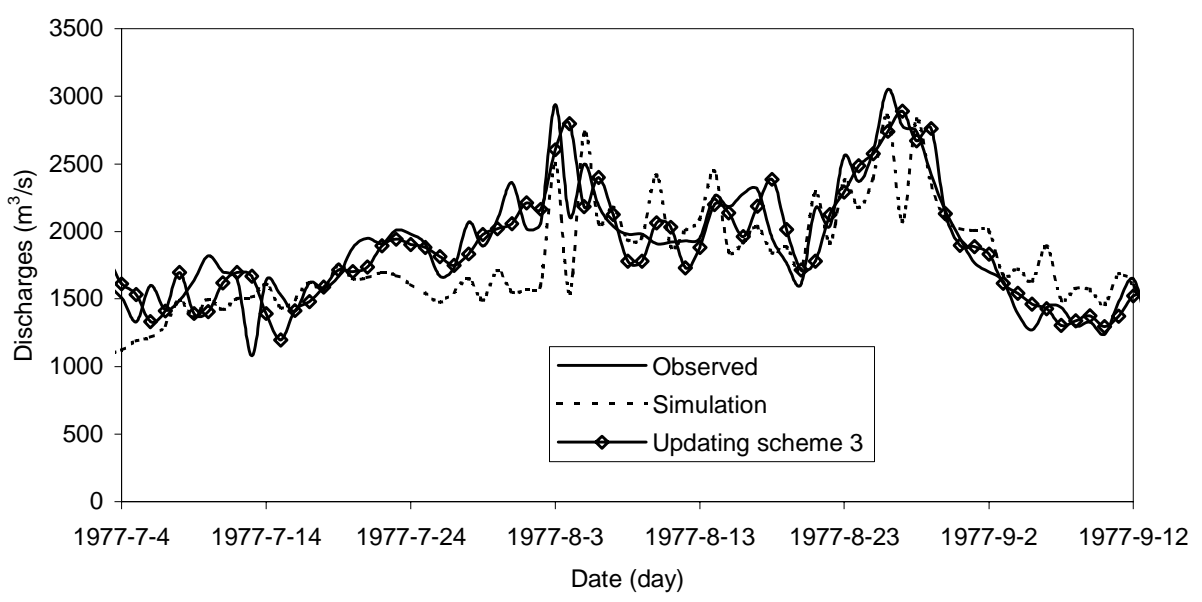

Fig.3. The simulated and observed hydrographs on the Sunkosi-1 catchment

of four weights are plotted in Figs. 5 to 8. Figure 5 plots the variation of the weight $v(1,1)$ and Fig. 6 of the weight $w(1,1)$, both on the Kizu catchment. Figure 7 is the plot of the variation of the weight $v(1,3)$ and Fig. 8 of the weight $w(3,1)$, both on the Shiquan-3 catchment,.

These four figures show that the variation in all four weights is small compared to its average value. For example, in the Kizu catchment, the weight $v(1,1)$ varies between 0.3445 and 0.3485 , while the weight $w(1,1)$ varies between 0.490 and 0.515 . In the Shiquan-3 catchment, the weight $v(1,3)$ varies between 0.150 and 0.162 , while the weight $w(3,1)$ varies between -2.0045 and -2.0020 . These changes 


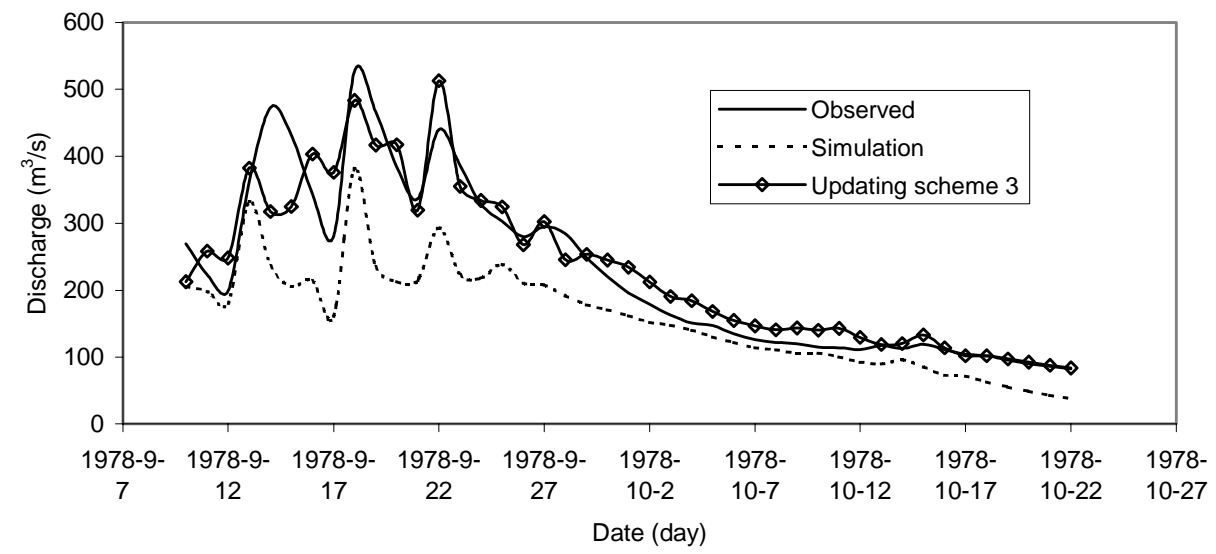

Fig.4. The simulated and observed hydrographs on the Yanbain catchment

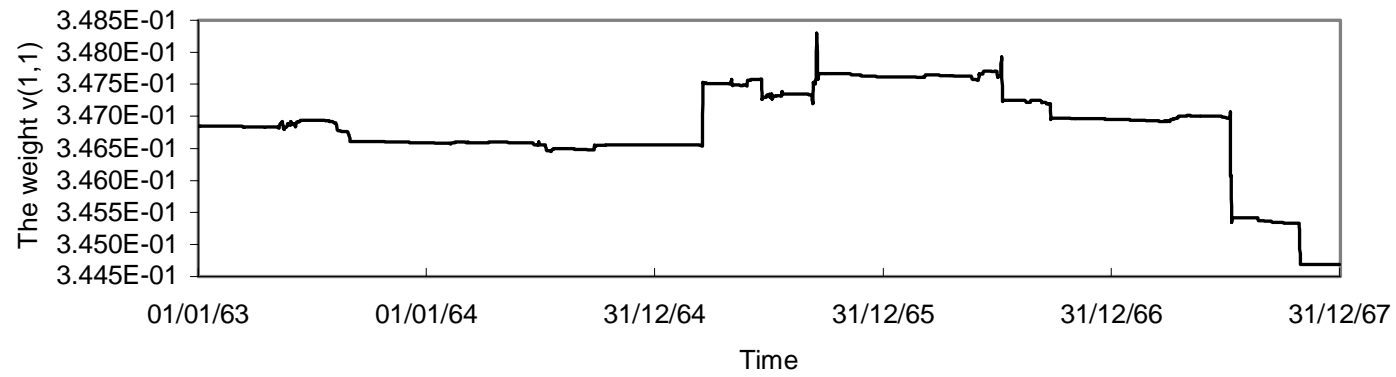

Fig.5. The variation of the weight $\mathrm{v}(1,1)$ in the third updating scheme on the Kizu catchment

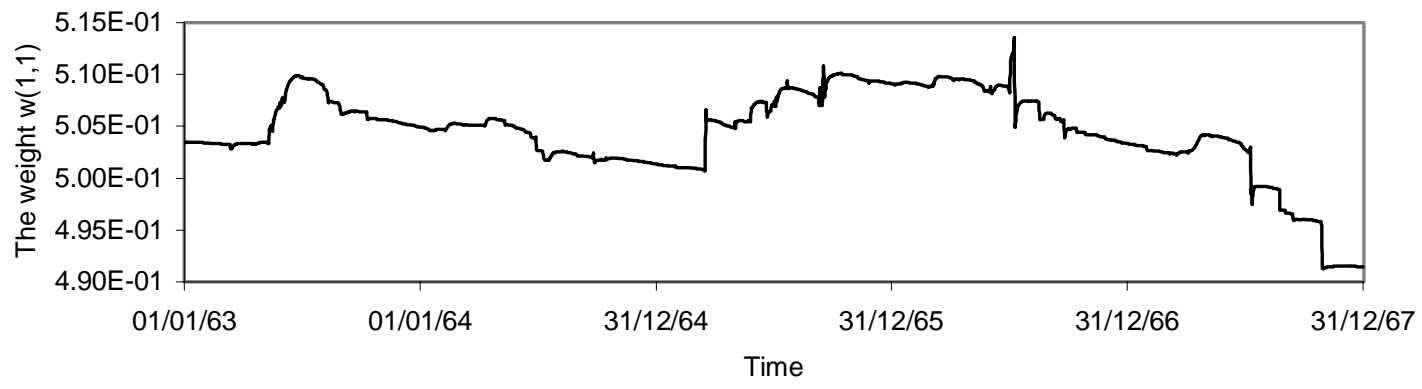

Fig. 6 The variation of the weight $\mathrm{w}(1,1)$ in the third updating scheme on the Kizu catchment

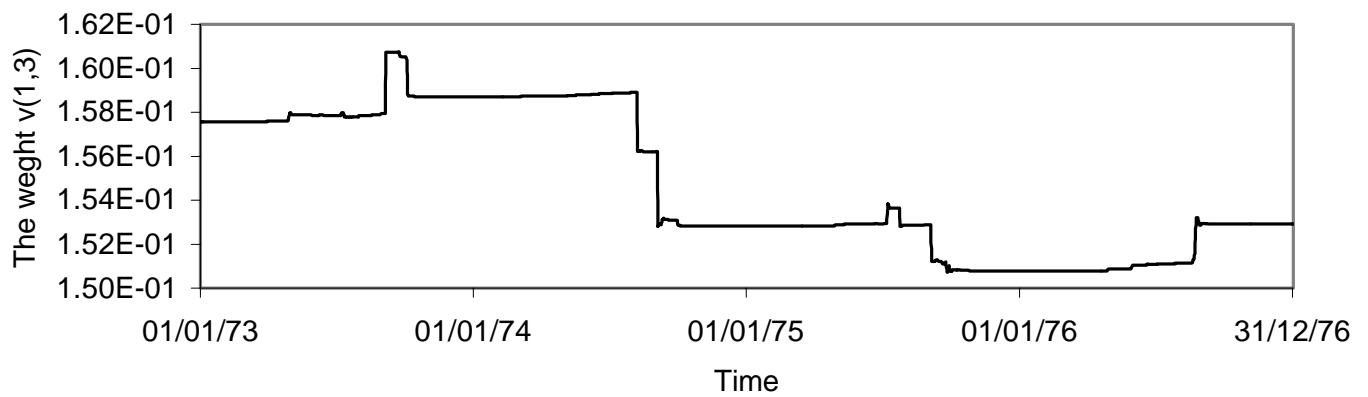

Fig.7. The variation of the weight $\mathrm{v}(1,3)$ in the third updating scheme on the Shiquan-3 catchment 


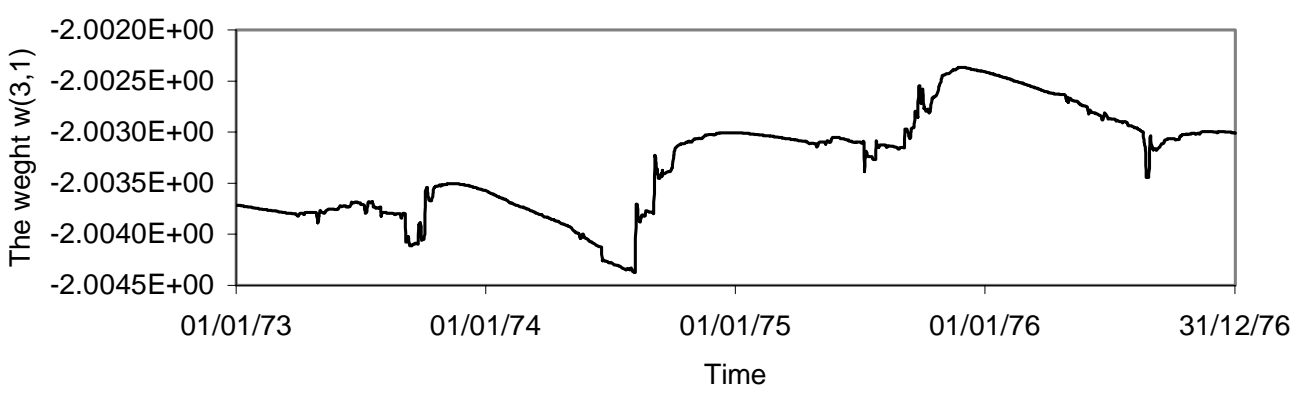

Fig. 8. The variation of the weight $\mathrm{w}(3,1)$ in the third updating scheme on the Shiquan-3 catchment

are small but even these small variations make the third updating scheme more effective than the first updating scheme, without any additional parameters.

\section{Discussions and conclusion}

In this paper, the three updating schemes using ANN in flow forecasting are compared in terms of model efficiency. The first updating scheme is the ANN model in the simulation mode plus an AR model. For the ANN model in the simulation model, the inputs include the observed rainfall and the previously estimated discharges, while the AR model is used to forecast the flow simulation errors of the ANN model. The second updating scheme is the ANN model in the updating mode, which uses the observed rather than the simulated discharges, together with observations of rainfall as the input, while the values of the weights fixed as the optimised values found by the Simplex method during the calibration period. The third updating scheme for flow forecasting is still the ANN model in the updating mode; however, the weights of the ANN model are no longer fixed but updated at each time step by the backpropagation method using the latest error in the forecast of the ANN model. Through updating the weights in the ANNs at the each step according to the backpropagation method, the outputs of the ANNs are also updated.

The results of comparing these three updating schemes in flow forecasting on the ten catchments show that the third updating scheme is more effective than the other two schemes in flow forecasting. In the first and second schemes, the parameters used in both the ANN (the substantive model) and the AR model (the minor model) are fixed and unable to reflect the time-varying characteristics of the hydrological processes in the catchment over rather long periods. In the third updating scheme, the continuous updating of all weights in the ANN according to the error in the forecast at the previous time step, enables the ANN model to track the time-variation characteristics of the hydrological processes. It is also more parsimonious in terms of the number of parameters; it does not need any correction model because the ANN self-adjusts its weights (or parameters) according to new information, when the backpropagation method is used to train the ANN model. Hence, this paper recommends that the ANN model with the backpropagation method, which updates the weights of ANN at each time step according to the latest forecast error, is used for real-time flow forecasting.

\section{Acknowledgments}

This research is supported by the Chinese National Key Basic Research Fund Pilot Project (2003CCA00200) and the Natural Sciences Fund of Hubei Province (2002AB009). The authors are very grateful to the editor and the referees for helpful comments on this paper.

\section{References}

ASCE Task Committee on definition of criteria for evaluation of catchment models of the Watershed Management Committee, Irrigation and Drainage Division, 1993. Criteria for evaluation of watershed models. J. Irrig. Drain. Engng., ASCE, 119, 429442.

Beran, M., 1999. Hydrograph prediction - How much skill? Hydrol. Earth Syst. Sci., 3, 305-308.

Campolo, M., Andreussi, P. and Soldati A., 1999. River flow forecasting with a neural network model. Water Resour. Res., 35, 1191-1197.

Campolo, M., Soldati, A. and Andreussi, P., 2003. Artificial neural network approach to flood forecasting in the River Arno. Hydrolog. Sci. J., 48, 381-398.

Chang, F. and Chen Y., 2001. A counterpropagation fuzzy-neural network modeling approach to real time streamflow prediction. J. Hydrol., 245, 153-164.

Chow, V. T., Maidment, D.R. and Mays, L.W., 1988. Applied Hydrology, McGraw-Hill, New York, USA. 202-203

Dekker, S.C., Bouten, W. and Schaap, M.G., 2001. Analysing forest transpiration model errors with artificial neural networks. $J$. Hydrol., 246, 197-208.

Duan, Q., Gupta, V.K. and Sorooshian, S., 1992. Effective and efficient global optimization for conceptual rainfall-runoff models. Water Resour. Res., 28, 1015-1031.

French, M.N., Krajewski, W.F. and Cuykendall, R.R., 1992. Rainfall forecasting in space and time using a neural network. J. Hydrol., 137, 1-31. 
Friedman, M. and Kandel, A., 1999. Introduction to Pattern Recognition: Statistical, Structural, Neural, and Fuzzy Logic Approaches, Series in Machine Perception Artificial Intelligence, vol. 32, World Scientific, Singapore.

Gautam, M.R., Watanabe, K. and Saegusa, H., 2000. Runoff analysis in humid forest catchment with artificial neural network. J. Hydrol., 235, 117-136.

Georgakakos, K.P. and Smith, G.F., 1990. On improved operational hydrologic forecasting: results from a WMO real-time forecasting experiment. J. Hydol., 114, 17-45.

Haykin, S., 1994. Neural Networks, MacMillan College Publishing Company, New York, USA.

Hsu, K.-L., Gupta, H.V. and Sorooshian, S., 1995. Artificial neural network modeling of the rainfall-runoff process. Water Resour. Res., 31, 2517-2530.

Imrie, C.E., Durucan, S. and Korre, A., 2000. River flow prediction using artificial neural networks: generalisation beyond the calibration range. J. Hydrol., 233, 138-153.

Jain, A., Sudheer, K. P. and Srinivasulu, S., 2004. Identification of physical processes inherent in artificial neural network rainfall runoff models. Hydrol. Process., 18, 571-581.

Kachroo, R.K. and Liang, G.C., 1992. River flow forecasting, part 2 , algebraic development of linear modelling techniques. $J$. Hydrol., 133, 17-40.

Legates, D.R. and McCabe Jr., G.J., 1999. Evaluating the use of "goodness-of-fit" measures in hydrologic and hydroclimatic model validation. Water Resour. Res., 35, 233-241.

Minns, A.W. and Hall, M.J., 1996. Artificial neural networks as rainfall-runoff models. Hydrolog. Sci. J., 41, 399-417.

Nash, J.E. and Sutcliffe, J., 1970. River flow forecasting through conceptual models. J. Hydrol., 10, 282-290.
Press, W.H., Flanney, B.P., Teukolsky, S.A. and Vetterling, W.T., 1989. Numerical Recipes, the Art of Scientific Computing, Cambridge University Press, UK. 312-326,

Rumelhart, D.E., Hinton, E. and Williams, J., 1986. Learning internal representation by error propagation. In: Parallel Distributed Processing, 1, 318-362. MIT Press, Cambridge, Mass.,USA.

Shamseldin, A.Y., 1997. Application of a neural network technique to rainfall-runoff modeling. J. Hydrol., 199, 272-294.

Shamseldin, A.Y. and O'Connor, K.M., 2001. A non-Linear neural network technique for updating river flow forecasts. Hydrol. Earth Syst. Sci., 5, 577-597.

Smith, J. and Eli, R.N., 1995. Neural network models of the rainfall-runoff process. J. Water Resour. Plan. Man.-ASCE, 121, 499-508.

Wilby, R.L., Abrahart, R.J. and Dawson, C.W., 2003. Detection of conceptual model rainfall-runoff processes inside an artificial neural network. Hydrolog. Sci. J., 48, 163-181.

WMO, 1975. Intercomparison of conceptual models used in operational hydrological forecasting (operational hydrology report No.7). World Meteorological Organization, Geneva, Switzerland.

WMO, 1992. Simulated Real-time Intercomparison of Hydrological Models (operational hydrology report No.38), World Meteorological Organization, Geneva, Switzerland. 814.

Xiong, L. and O'Connor, K.M., 2002. Comparison of four updating models for real-time river flow forecasting. Hydrolog. Sci. J., 47, 621-639.

Zhu, M.L., Fujita, M. and Hashimoto, N., 1994. Application of neural networks to runoff prediction. In: Stochastic and Statistical Methods in Hydrology and Environmental Engineering, K.W.Hipel et al. (Eds.).Kluwer, Dordrecht, The Netherlands. 205-216 . 